\section{Collective housing of social mixture in downtown La Serena, Chile}

PALABRAS CLAVE | VIVIENDA COLECTIVA | INTECRACIÓN SOCIAL I DERECHO A LA CIUDAD

KEYWORDS | COLLECTIVE HOUSING | SOCIAL INTECRATION IRICHT TO THE CITY

\section{| RESUMEN |}

El mayor problema hoy en Chile es su desigualdad. Indicadores de la OCDE lo sitúan como uno de los países de mayor desigualdad urbana. Este proyecto propone cambios tanto de gestión urbana como de arquitectura y mixtura tipológica de conjuntos habitacionales. La idea es revitalizar el centro histórico de La Serena, promoviendo viviendas colectivas en densidad media considerando la población preexistente, en su mayoría allegados, evitando la gentrificación y densificando el sector con diversas tipologías y equipamiento. A nivel de gestión urbana, se propone un sistema de Empresa Pública que promueva la integración y equipamientos de escala intermedia. Siendo la integración lo fundamental, a nivel arquitectónico, el conjunto contempla tres escalas de integración: comunal, barrial y vecinal. Así, se plantea la construcción de un parque comunal aledaño al polígono del conjunto. Además, se proyectan en primer piso del edificio una amplia plaza verde que se fuga con vista al Río Elqui una plaza dura contenida de carácter interior y comunitario. Finalmente, cada piso superior contiene uno o dos espacios de integración en los vértices del volumen que conectados a través de amplias galerías promueven la interacción de las siete diversas tipologías y 150 viviendas que componen esta propuesta.

\section{| ABSTRACT |}

The main problem in Chile today is inequality. Regional and OECD's research indicates that Chile has one of the highest urban segregation levels. This project proposes changes in both, urban management and architecture, and a typological mix of collective housing. The idea is to revitalize the historic downtown area of La Serena by promoting collective housing of medium density, considering the preexisting inhabitants, mostly "allegados", thus avoiding gentrification and, at the same time, increasing density through diverse typologies and infrastructure. On an urban management level, a system of public enterprise that promotes integration and projects of intermediate scale is proposed, beino integration most fundamental. On an architectural level, the housing complex considers three scales of integration: city, neighborhood and community.

\title{
Conjunto de integración y mixtura social en el centro de La Serena, Chile*
}

\author{
FERNANDO TORO CANO**·Santiago, Chile·fernandotorocano@gmail.com
}

\section{PROBLEMÁTICA}

El principal problema de Chile y América Latina hoy es su desigualdad. Indicadores tanto regionales como de la OCDE demuestran cómo en las últimas décadas este país ha sido uno de los que lidera los rankings de dicha problemática. El elevado Índice de Gini se materializa en ciudades cada vez más segregadas y en condiciones urbanas contrastantes entre los distintos sectores de la ciudad. Las áreas verdes, el espacio público y los equipamientos de calidad se ubican en los sectores de mayores ingresos y la vivienda social y las áreas deterioradas quedan relegadas a la periferia debido a la falta de regulación de parte del Estado y a la falta de una política de suelo, afectando directamente no solo a los sectores más vulnerables, sino que también al Estado, que ha debido invertir importantes recursos económicos y humanos para solucionar este déficit.
Se han generado diversas políticas públicas para apoyar esta problemática, entre ellas el programa "Quiero Mi Barrio", los proyectos de integración social, el subsidio a la localización e incluso ahora últimamente el subsidio de arriendo, que si bien han tenido resultados dispares, todos aparecen como políticas momentáneas o de turno y no basales. Este proyecto entonces propone un cambio fundamental en la gestión urbana actual, atribuyendo mayor responsabilidad a los gobiernos locales y la posibilidad de constituir empresas públicas cuyo director ejecutivo sea nombrado por la municipalidad. Así, tomando como ejemplo la empresa pública de Madrid y Medellín, ambas de excelentes resultados al día de hoy, se genera esta figura que tiene como objetivo desarrollar proyectos de mediana y gran escala, cuyo fin último sea la integración y la mixtura social.

Artículo en base a los resultados del proyecto de título "Conjunto de integración y mixtura social en el centro de Lo Serena" realizado por el autor y presentado en enero de 2015 para obtener el grado de Arquitecto en la Facultad de Arquitectura y Urbanismo de la Universidad de Chile. Profesores guía: Humberto Eliash y Sebastián Lambiassi.

** Arquitecto (2015) y estudiante del Magíster en Dirección y Administración de Proyectos Inmobiliarios, ambos en la Facultad de Arquitectura y Urbanismo de la Universidad de Chile. 
1. Vista acceso. Elaboración propia.

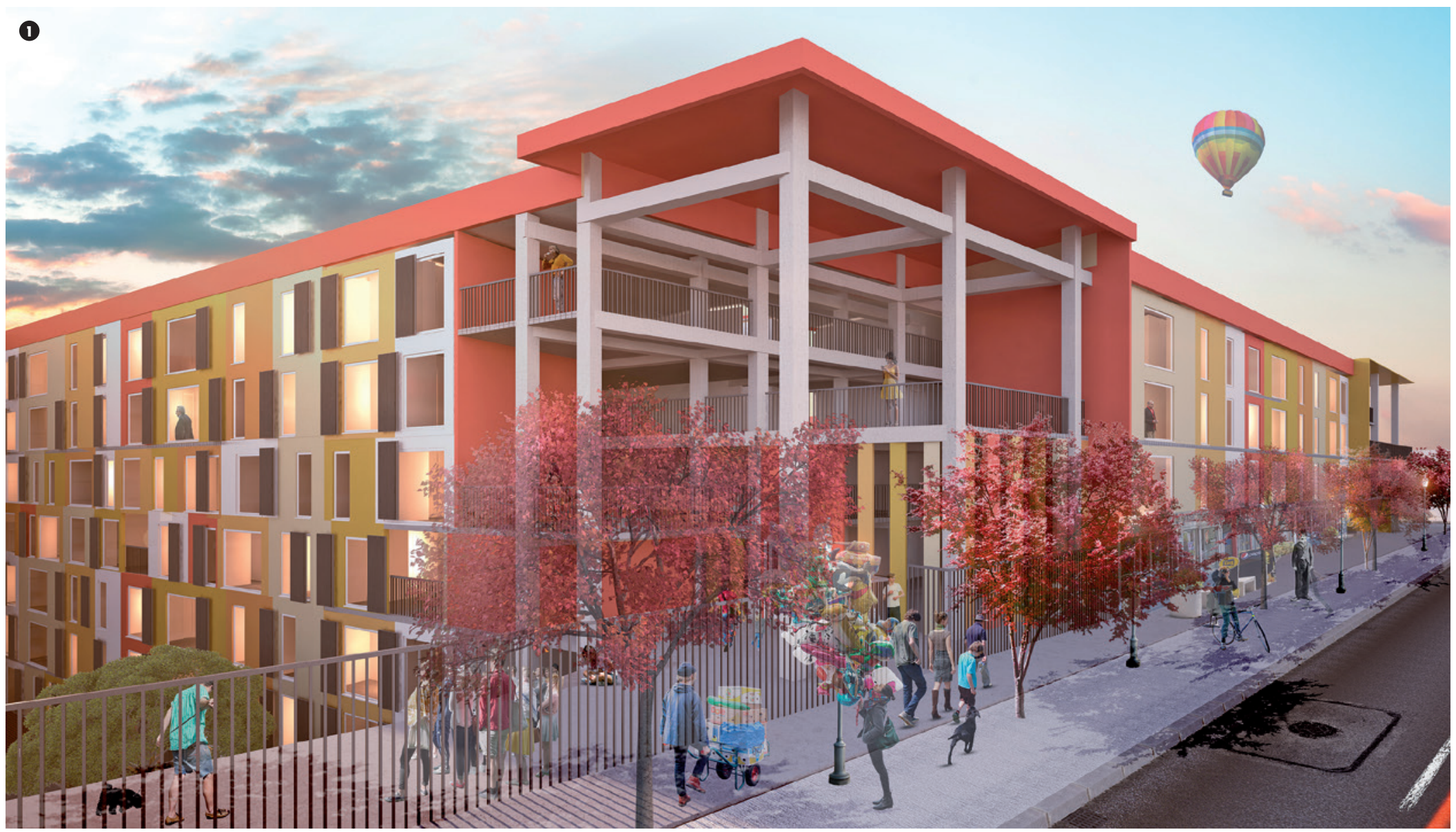

\section{UBICACIÓN, MODELO DE GESTIÓN Y MIXTURA}

El proyecto emplazado en el borde del centro histórico de La Serena y a orillas del Río Elqui, comienza con la premisa de trabajar sectores centrales de ciudades intermedias, es decir, ciudades entre 50.000 y 350.000 habitantes, aún no consolidadas completamente donde el valor de suelo aún permite alguna mínima intervención estatal en sectores bien ubicados.
El conjunto se sitúa en un paño mixto, público y privado, que por el lado de la ribera pertenece a Bienes Nacionales y por el lado urbano a alrededor de 40 propietarios de escasos recursos y que posiblemente se vean forzados a vender su propiedad (gentrificación) por el nuevo Plan Regulador Comunal ad portas de ser aprobado, y que permite la construcción de elevadas edificaciones destinadas a sectores medios y altos.
Basado en el Derecho a la Ciudad y considerando el modelo de gestión propuesto, mediante empresas municipales, este proyecto que aborda cuatro manzanas y desarrolla solo una, propone la construcción de dos edificios de vivienda (300 en total) bajo el sistema de proyectos de integración social, y dos manzanas de equipamiento de escala intermedia: un museo interactivo tecnológico y un parque de escala comunal. 
2. Vista aérea. Elaboración propia.

3. Estrategias de diseño. Elaboración propia.
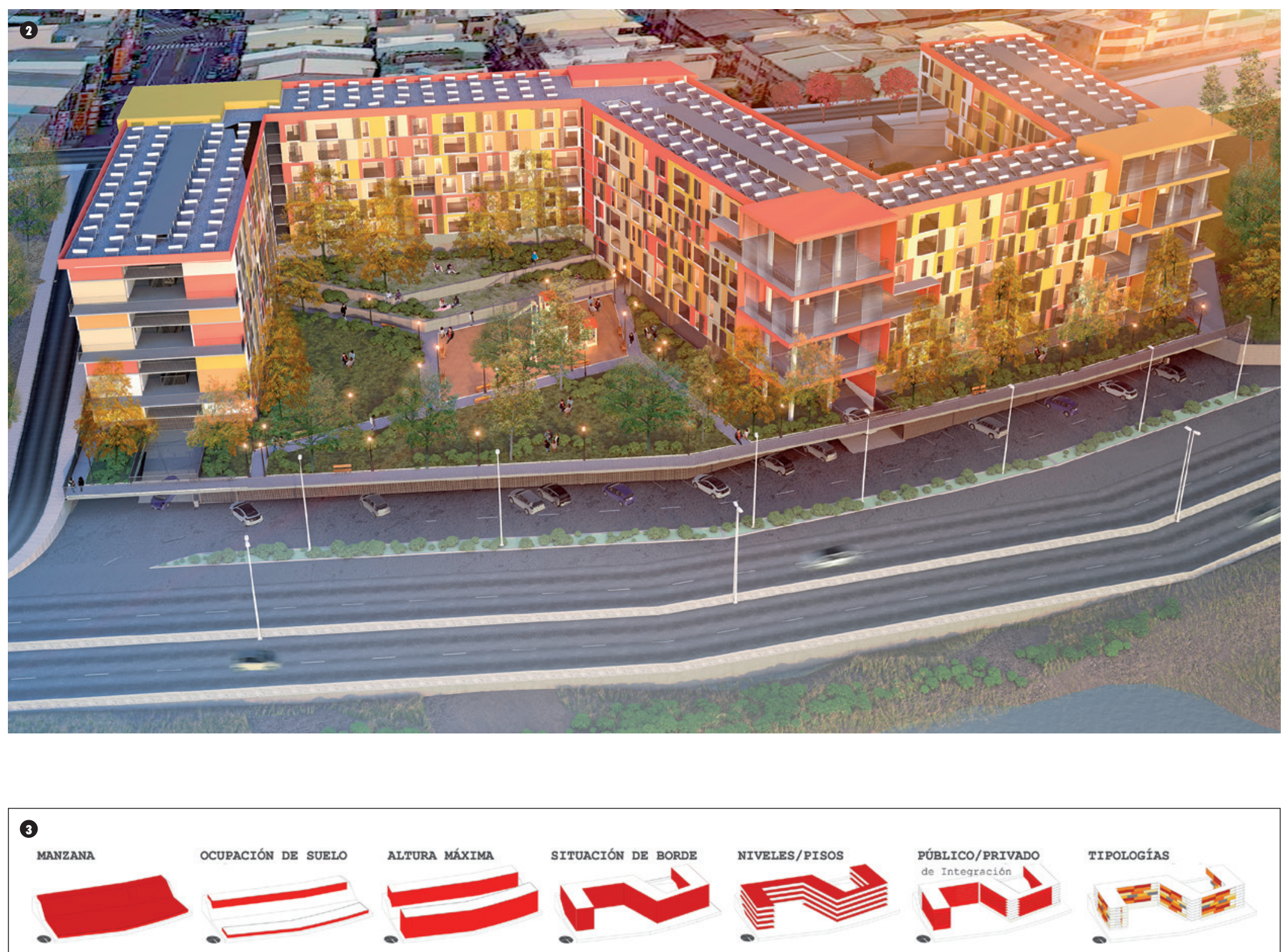
4. Planta $5^{\text {to }}$ nivel. Elaboración propia.

(4)

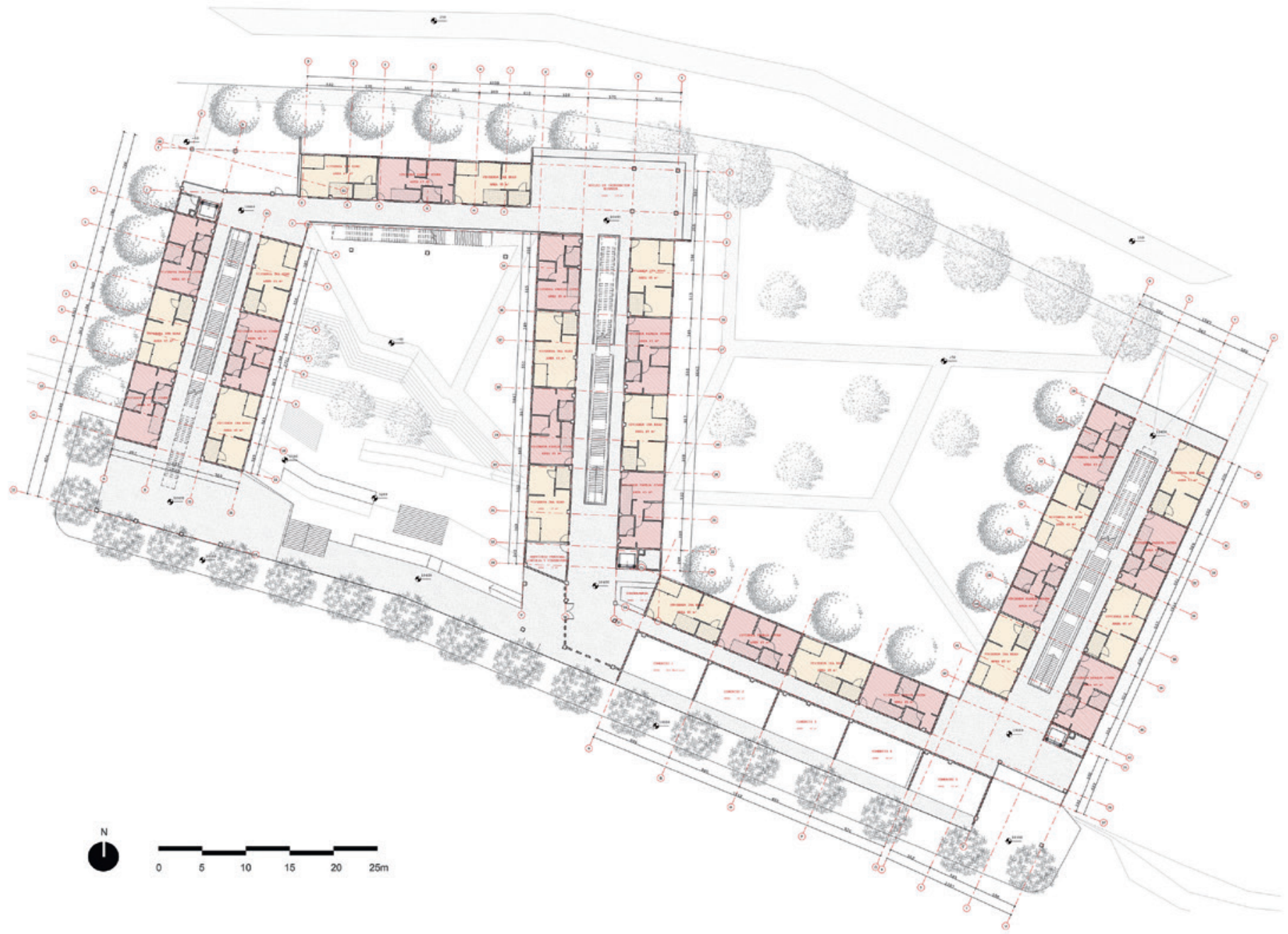

\section{PROPUESTA VOLUMÉTRICA Y TIPOLOGÍAS}

El edificio proyectado considera las dos situaciones preexistentes de límite, río y ciudad, y los aspectos bioclimáticos del terreno, así se crea la planta con forma de S, cobijando dos patios de distinta calidad espacial y funcional. Por un lado tenemos un patio duro de carácter comunitario notoriamente más contenido y destinado para celebraciones y actos de la comunidad y otro, más verde que reconoce la fuga que entrega el edificio hacia el río Elqui generando un diseño paisajístico con vegetación y dispositivos de bajo consumo energético e hídrico. Considerando la normativa del paño, se divide la altura máxima en 8 pisos generando barrios en altura, ya que en cada piso existe un promedio de 19 viviendas y espacios semipúblicos, situados en los vértices de la $S$, que poseen un espacio comunitario destinado específicamente a responder necesidades sociales del carácter de cada piso (jóvenes, familias, tercera edad, etc.).

Cada piso está determinado por tres espacios fundamentales: viviendas en doble crujía, los espacios públicos bisagra o de mixtura social (sala de estudio, miradores, máquinas de ejercicio al aire libre, etc.) y una galería o pasillo central de 2,5 metros de ancho que 
5

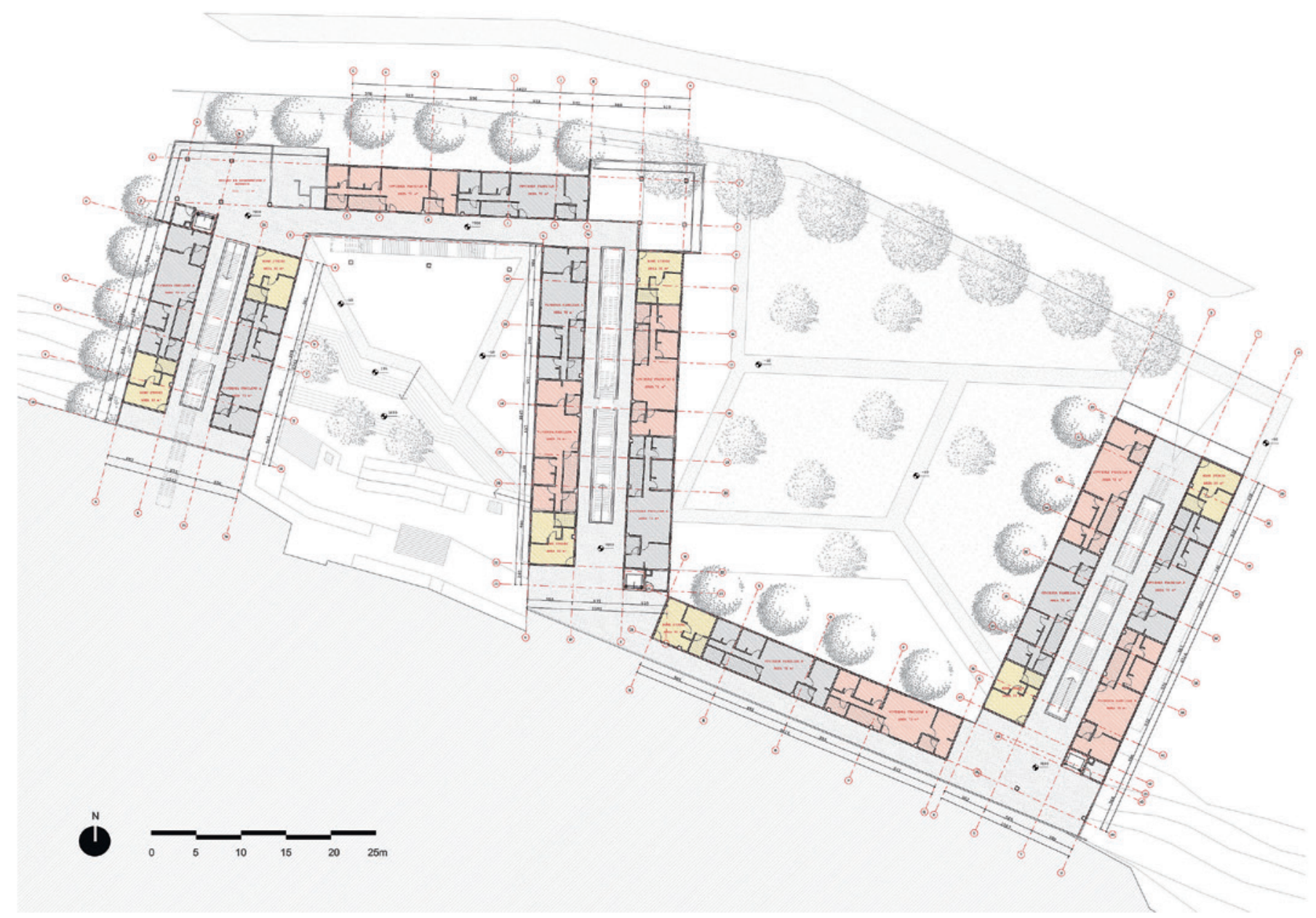

contiene la circulación vertical. A nivel social este aspecto es muy importante, ya que se diagnostica que los pasillos con poco ancho son utilizados netamente para la circulación, en cambio, pasillos con mayor espacio, dan la posibilidad de sociabilizar y realizar otras actividades. A nivel funcional, estos pasillos abiertos en la techumbre y en sus extremos dan la posibilidad de tener una constante iluminación y ventilación cruzada tanto a nivel de edificio como de vivienda, disminuyendo notoriamente los requerimientos energéticos del conjunto. Las siete tipologías propuestas (ver figura 6) en las más de 150 viviendas, y los regímenes de venta (subsidios y privados) y renta (empresa pública) de los diversos departamentos entregan al conjunto un dinamismo necesario para la mixtura y la integración social. El edificio comprende así un experimento a nivel de gestión urbana, diseño arquitectónico y aspectos sociales, definiendo relaciones públicas a escala comunal, barrial y vecinal con el parque, el primer piso y los espacios de integración respectivamente. Sometido a una evaluación económica considerando rentabilidad social, este proyecto es una propuesta con una visión largo plazo, ya que se estima que la rentabilidad estimada se lograría en 20 años, algo que creo necesario considerar si queremos avanzar en una deuda habitacional hoy cortoplacista. 
6. Esquema de tipologías. Elaboración propia.

(0

TIPOLOGÍAS = Normativa - Crecimiento demográfico - Rentabilidad social

,

ESTUDIANTE

PAREJA JOVEN

MOVILIDAD REDUCIDA

$1 \mathrm{~B}$

$1 \mathrm{C}$

$1 \mathrm{C}$

$1 \mathrm{~L} / \mathrm{C}$

$1 \mathrm{~L} / \mathrm{C}$

es $\$ 180.000$

$\$ 200.000$

$45 \mathrm{M} 2$

긴 30M2

Arriendo

Subsidio de Arriendo

范

量
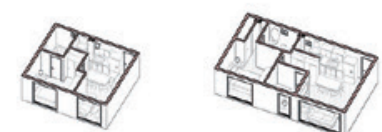

告号

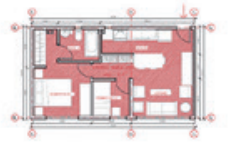

葸突 $10 \%$

$10 \%$

$10 \%$ $\dot{c} / \mathrm{f}$

iti

1D

$1 \mathrm{~B}$

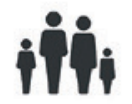

$3 D$
$2 B$
$1 C$
$1 L / C$

UF1. 000

63M2

Subsidio DS 1 Tit.1
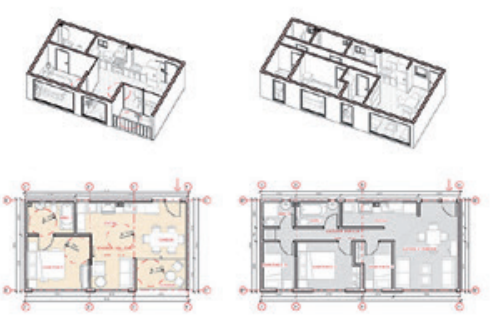

FAMILIA VULNERABLE

FAMILIA EMERGENTE
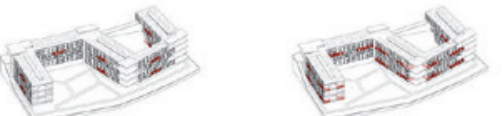

$20 \%$
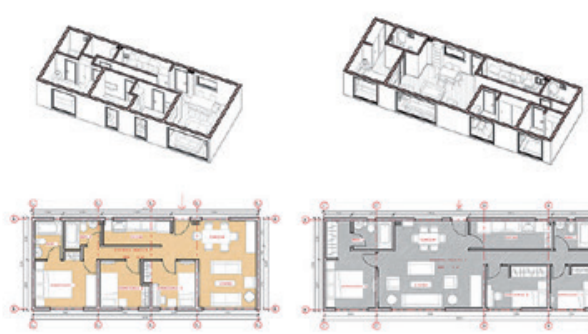

$22 \%$

$14 \%$
FAMILIA EMERGENTE
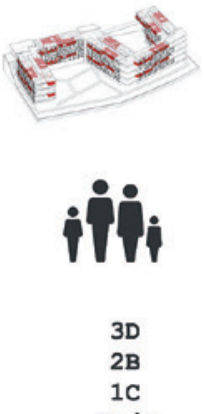

1L/C

UF1. 600

75M2

Subsidio

DS 1 Tít. 2

FAMILIA CLASE MEDIA

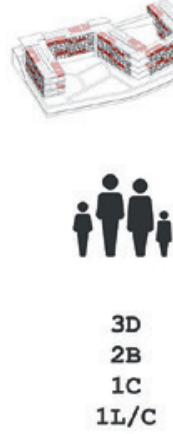

UF2. 000

75M2

$$
\text { Sin }
$$
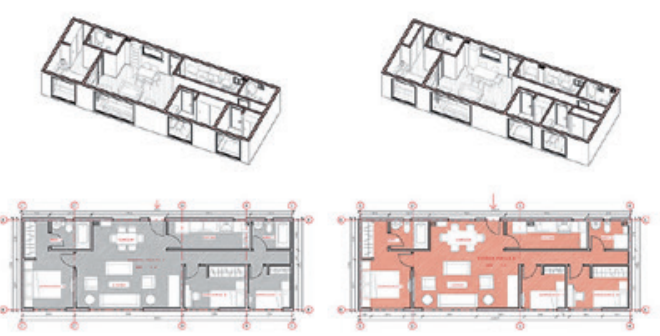

$14 \%$

\section{ASPECTOS GENERALES Y TECNOLÓGICOS}

A nivel tecnológico, el edificio cuenta con una dotación de 170 colectores solares en techumbre transitable, cuyo fin último es disminuir los gastos por conceptos de agua caliente. Además, y bajo la colaboración de privados, se diseñan paneles exteriores deslizables con una trama vertical u horizontal según orientación, que responden a un módulo determinado de 30, 60 o $90 \mathrm{~cm}$ y que protegen del crítico soleamiento en La Serena. Las viviendas están diseñadas con una cruía de 6 metros que divide a través de un pasillo dos zonas: habitables y húmedas. La logia no se considera, ya que se dispone además de un jardín, una lavandería de uso y mantención comunitaria en los pisos inferiores, esto, entendiendo que actualmente uno de los mayores problemas de la vivienda económica actual es el deterioro por conceptos de humedad y de utilización de balcones para fines de secado y bodegaje, dando posibilidad de abalconamiento solo a las viviendas para movilidad reducida, también incluidas en el proyecto. 
7. Fachada norte. Elaboración propia.

8. Patio comunitario. Elaboración propia.

9. Vista interior núcleo integración mirador. Elaboración propia.
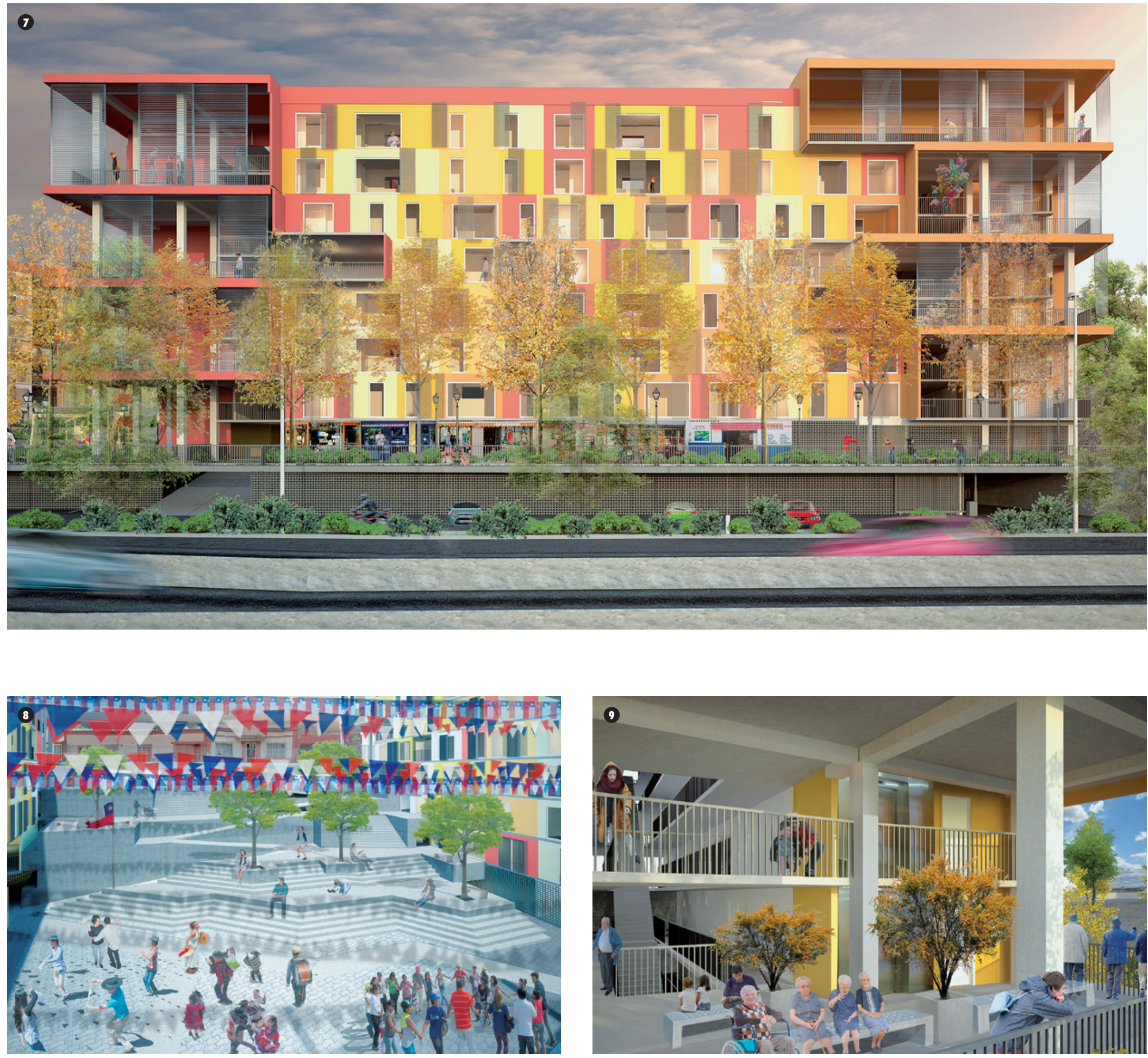